\title{
Rough Sets in Perception-Based Computing
}

\section{Extended Abstract}

\author{
Andrzej Skowron \\ Institute of Mathematics, Warsaw University, \\ Banacha 2, 02-097 Warsaw, Poland \\ skowron@mimuw. edu.pl
}

Intelligent systems for many real life problems can be modeled by systems of complex objects and their parts changing and interacting over time. The objects are usually linked by certain dependencies, can cooperate between themselves and are able to perform complex and flexible actions (operations) in an autonomous manner. Such systems are identified as complex dynamical systems [240], autonomous multiagent systems [2040], or swarm intelligent systems (see, e.g., 2817).

One of the main challenges to be solved in intelligent systems research is the development of methods for approximate reasoning from measurements to perception, i.e., from concepts that can be directly approximated using sensor measurements to concepts, expressed by human beings in natural language, that are the perception results 42 .

The existing methodologies and technologies are not adequate to solve many problems associated with this challenge. Among such problems are, e.g., classification and understanding of medical images [30], control of autonomous systems such as unmanned aerial vehicles or robots (see, e.g., [47/44]) or problems pertaining to monitoring or rescue tasks in multiagent systems [11].

Nowadays, new emerging computing paradigms are investigated in an attempt to develop methods for solving such problems. The further progress depends on a successful cooperation of specialists from different scientific disciplines such as mathematics, logic, philosophy, computer science, artificial intelligence, biology, physics, chemistry, bioinformatics, medicine, neuroscience, linguistics, psychology, and sociology. In particular, different aspects of reasoning from measurements to perception are investigated in psychology [1316, neuroscience [30 24], theories of cognition [21], layered learning [39], mathematics of learning [30, machine learning, pattern recognition [14, data mining [17. and also by researchers working on recently emerged computing paradigms, like computing with words and perception [43], granular computing [25], rough sets, rough mereology, and rough-neural computing [25].

In this lecture we overview some of these new computing paradigms and some of the interactions between the various disciplines that have been mentioned.

The concept approximation problem is the basic problem investigated in machine learning, pattern recognition [14 and data mining [17]. It is necessary to induce approximations of concepts (models of concepts) from available experimental data. The data models developed so far in such areas as statistical 
learning, machine learning, pattern recognition are not satisfactory for approximation of complex concepts that occur in the perception process. Researchers from the different areas have recognized the necessity to work on new methods for concept approximation (see, e.g., 841]). The main reason for this is that these complex concepts are, in a sense, too far from measurements which makes the searching for relevant features infeasible in a very huge space. There are several research directions aiming at overcoming this difficulty. One of them is based on the interdisciplinary research where the knowledge pertaining to perception in psychology or neuroscience is used to help to deal with complex concepts (see, e.g., [30]). There is a great effort in neuroscience towards understanding the hierarchical structures of neural networks in living organisms [1230. Also mathematicians are recognizing problems of learning as the main problem of the current century [30. These problems are closely related to complex system modeling as well. In such systems again the problem of concept approximation and its role in reasoning about perceptions is one of the challenges nowadays. One should take into account that modeling complex phenomena entails the use of local models (captured by local agents, if one would like to use the multiagent terminology [20]) that should be fused afterwards. This process involves negotiations between agents 20] to resolve contradictions and conflicts in local modeling. This kind of modeling is becoming more and more important in dealing with complex real-life phenomena which we are unable to model using traditional analytical approaches. The latter approaches lead to exact models. However, the necessary assumptions used to develop them result in solutions that are too far from reality to be accepted. New methods or even a new science therefore should be developed for such modeling [15].

One of the possible approaches in developing methods for complex concept approximations can be based on the layered learning [39]. Inducing concept approximation should be developed hierarchically starting from concepts that can be directly approximated using sensor measurements toward complex target concepts related to perception. This general idea can be realized using additional domain knowledge represented in natural language. For example, one can use some rules of behavior on the roads, expressed in natural language, to assess from recordings (made, e.g., by camera and other sensors) of actual traffic situations, if a particular situation is safe or not [22]. To deal with such problems one should develop methods for concept approximations together with methods aiming at approximation of reasoning schemes (over such concepts) expressed in natural language. The foundations of such an approach, creating a core of perception logic, are based on rough set theory [27] and its extension rough mereology 3125, both invented in Poland, in combination with other soft computing tools, in particular with fuzzy sets.

Rough set theory due to Zdzisaw Pawlak [27, is a mathematical approach to imperfect knowledge. The problem of imperfect knowledge has been tackled for a long time by philosophers, logicians and mathematicians. Recently it became also a crucial issue for computer scientists, particularly in the area of artificial intelligence. There are many approaches to the problem of how to understand 
and manipulate imperfect knowledge. The most successful one is, no doubt, the fuzzy set theory proposed by Lotfi A. Zadeh [42]. Rough set theory presents still another attempt to solve this problem. It is based on an assumption that objects are perceived by partial information about them. Due to this some objects can be indiscernible. From this fact it follows that some sets can not be exactly described by available information about objects; they are rough not crisp. Any rough set is characterized by its (lower and upper) approximations. The difference between the upper and lower approximation of a given set is called its boundary. Rough set theory expresses vagueness by employing a boundary region of a set. If the boundary region of a set is empty it means that the set is crisp, otherwise the set is rough (inexact). A nonempty boundary region of a set indicates that our knowledge about the set is not sufficient to define the set precisely. One can recognize that rough set theory is, in a sense, a formalization of the idea presented by Gotlob Frege [13.

One of the consequences of perceiving objects using only available information about them is that for some objects one cannot decide if they belong to a given set or not. However, one can estimate the degree to which objects belong to sets. This is another crucial observation in building foundations for approximate reasoning. In dealing with imperfect knowledge one can only characterize satisfiability of relations between objects to a degree, not precisely. Among relations on objects the rough inclusion relation, which describes to what degree objects are parts of other objects, plays a special role. A rough mereological approach (see, e.g., [313525]) is an extension of the Leśniewski mereology [19] and is based on the relation to be a part to a degree. It will be interesting to note here that Jan Łukasiewicz was the first who started to investigate the inclusion to a degree of concepts in his discussion on relationships between probability and logical calculi [18.

The very successful technique for rough set methods was Boolean reasoning 9. The idea of Boolean reasoning is based on construction for a given problem $P$ a corresponding Boolean function $f_{P}$ with the following property: the solutions for the problem $P$ can be decoded from prime implicants of the Boolean function $f_{P}$. It is worth to mention that to solve real-life problems it is necessary to deal with Boolean functions having a large number of variables.

A successful methodology based on the discernibility of objects and Boolean reasoning has been developed in rough set theory for computing of many key constructs like reducts and their approximations, decision rules, association rules, discretization of real value attributes, symbolic value grouping, searching for new features defined by oblique hyperplanes or higher order surfaces, pattern extraction from data as well as conflict resolution or negotiation 32. Most of the problems involving the computation of these entities are NP-complete or NPhard. However, we have been successful in developing efficient heuristics yielding sub-optimal solutions for these problems. The results of experiments on many data sets are very promising. They show very good quality of solutions generated by the heuristics in comparison with other methods reported in literature (e.g., with respect to the classification quality of unseen objects). Moreover, they are 
very time-efficient. It is important to note that the methodology makes it possible to construct heuristics having a very important approximation property. Namely, expressions generated by heuristics (i.e., implicants) close to prime implicants define approximate solutions for the problem (see, e.g., [46]).

A question arises if the methods developed so far based on Boolean and approximate Boolean reasoning can be scaled to the case of complex problems considered here.

Rough set theory has attracted attention of many researchers and practitioners all over the world, who have contributed essentially to its development and applications. The rough set approach seems to be of fundamental importance to AI and cognitive sciences, especially in the areas of machine learning, knowledge acquisition, decision analysis, knowledge discovery from databases, expert systems, inductive reasoning and pattern recognition.

The rough set approach can be used in searching for complex patterns relevant for approximation of vague concepts. Different stages of this searching process are strongly related to perception.

Let us consider two simple illustrative examples supporting this claim.

In searching for relevant features often a function $f$, transforming objects from a given set $U$ into simpler objects from another set $U_{0}$, is defined. In image or signal analyzis, objects from $U$ are represented by relational structures while objects from $U_{0}$ are parts of objects from $U$ or some other relational structures. The selection of relevant transformations $f$ can be interpreted as the relevant structural perception of objects or their parts. Next, a set $A$ of features (attributes) is defined on $U_{0}$. Any feature (attribute) $a$ is a function from $U_{0}$ into $V_{a}$, where $V_{a}$ is the value set of $a$. The indiscernibility relation $I N D(B) \subseteq U_{0} \times U_{0}$ for $B \subseteq A$ [27] can be extended to the indiscernibility relation $I N D^{*}(B, f) \subseteq U \times U$ by $u I N D^{*}(B, f) u^{\prime}$ if and only if $f(u) I N D(B) f\left(u^{\prime}\right)$. Hence, the indiscernibility classes of $I N D^{*}(B, f)$ can be adjusted using parameters $f$ and $B$. However, the search space for $f$ and $B$ is very large. Therefore, some hints from research on perception that would make this search more efficient will be of great importance [6].

Perception of relevant context for a given object is an important task in pattern recognition of complex objects. To see how such a context can be defined in the rough set approach, let us consider slightly modified information systems [27. We assume that for any attribute $a$ not only the value set $V_{a}$ is given but also a relational structure $\mathcal{R}_{a}$ over $V_{a}$. One can define neighborhoods over such relational structures. Such neighborhoods can be sets defined by formulas interpreted in $\mathcal{R}_{a}$ or some substructures of $\mathcal{R}_{a}$. For example, one can define in this way time windows or some neighborhoods over time windows representing relational structures over time windows. Next, such neighborhoods can be fused and used to induce neighborhoods of objects [36. The searching process for relevant object neighborhoods and their properties is computationally expensive. Hence, again some hints from research on perception can help to make this process more efficient. 
There are many more examples linking the research on rough sets with the research on perception. For example, reasoning about changes [37, or relevant granulation of complex objects (information granules) [29.

Further cooperation between researchers working in different areas on perception can lead to advancement in constructing more efficient algorithms involved in searching for complex patterns and corresponding to different stages of the perception processes.

In a more general setting, objects we are dealing with are information granules. Such granules are obtained as the result of information granulation (see, e.g., 43[33|35|25]). Information granulation can be viewed as a human way of achieving data compression and it plays a key role in implementing the divideand-conquer strategy in human problem-solving [43].

Computing with Words and Perceptions "derives from the fact that it opens the door to computation and reasoning with information which is perception rather than measurement-based. Perceptions play a key role in human cognition, and underlie the remarkable human capability to perform a wide variety of physical and mental tasks without any measurements and any computations. Everyday examples of such tasks are driving a car in city traffic, playing tennis and summarizing a story" 43.

The rough mereological approach (see, e.g., 31|25]) is based on calculi of information granules for constructing complex concept approximations. Constructions of information granules should be robust with respect to their input information granule deviations. Hence, the information granule construction process itself can also be granulated. As the result the so called AR schemes (AR networks) [31 32 33 35] are obtained. AR schemes can be interpreted as complex patterns [17. Searching methods for such patterns relevant to a given target concept have been developed [35. Methods for deriving relevant AR schemes are computationally expensive. This complexity can be substantially reduced by using domain knowledge. In such a case AR schemes are derived in conformity with reasoning schemes in natural language that are ellicited from domain knowledge. Developing methods for deriving such AR schemes is one of the main goals of our current projects.

The ontology approximation problem is one of the fundamental problems related to approximate reasoning in distributed environments [38|34]. One should construct (in a given language that is different from the ontology specification language) not only approximations of concepts from ontology but also vague dependencies specified in the ontology. It is worthwhile to mention that ontology approximation should be induced on the basis of incomplete information about concepts and dependencies specified in the ontology. Information granule calculi based on rough sets have been proposed as tools making it possible to solve this problem.

We discuss ontology approximation in the granular computing framework. In particular, approximation of any vague dependency is a method which allows any object to compute the arguments "for" and "against" its membership to the dependency conclusion on the basis of the analogous arguments 
relative to the dependency premisses. Any argument is a compound information granule (compound pattern). Arguments are fused by local schemes (production rules) discovered from data. Further fusions are possible through composition of local schemes, called approximate reasoning schemes (AR schemes) (see, e.g., 3143335). To estimate the degree to which (at least) an object belongs to concepts from an ontology the arguments "for" and "against" those concepts are collected and next a conflict resolution strategy is applied to them for predicting the degree. Several information granule calculi are involved in solving the problem of ontology approximation. Information granules are represented by compound patterns. By granulation of the discovered patterns to layers of vague concepts one can obtain more relevant approximations of dependencies by using the rough-fuzzy approach based on granulation.

Let us mention some illustrative examples related to our current projects in which ontology approximation is involved.

The prediction of behavioral patterns of a complex object evaluated over time is usually based on some historical knowledge representation used to store information about changes in relevant features or parameters. This information is usually represented as a data set and has to be collected during long-term observation of a complex dynamic system. For example, in case of road traffic, we associate the object-vehicle parameters with the readouts of different measuring devices or technical equipment placed inside the vehicle or in the outside environment (e.g., alongside the road, in a helicopter observing the situation on the road, in a traffic patrol vehicle). Many monitoring devices serve as informative sensors such as GPS, laser scanners, thermometers, range finders, digital cameras, radar, image and sound converters (see, e.g. [40]). Hence, many vehicle features serve as models of physical sensors. Here are some exemplary sensors: location, speed, current acceleration or deceleration, visibility, humidity (slipperiness) of the road. By analogy to this example, many features of complex objects are often dubbed sensors. In the lecture we discuss (see also [5]) some rough set tools for perception modelling that make it possible to recognize behavioral patterns of objects and their parts changing over time. More complex behaviour of complex objects or groups of complex objects can be presented in the form of behavioral graphs. Any behavioral graph can be interpreted as a behavioral pattern and can be used as a complex classifier for recognition of complex behaviours. We outline [5] the complete approach to the perception of behavioral patterns, that is based on behavioral graphs and the dynamic elimination of behavioral patterns. The tools for dynamic elimination of behavioral patterns are used for switching-off in the system attention procedures searching for identification of some behavioral patterns. The developed rough set tools for perception modeling are used to model networks of classifiers. Such networks make it possible to recognize behavioral patterns of objects changing over time. They are constructed using an ontology of concepts provided by experts that engage in approximate reasoning on concepts embedded in such an ontology. Experiments on data from a vehicular traffic simulator [45] are showing that the developed methods are useful in the identification of behavioral patterns. 
Our second example concerns human computer-interfaces that allow for a dialog with experts to transfer to the system their knowledge about structurally complex objects. For pattern recognition systems [10], e.g., for Optical Character Recognition (OCR) systems it will be helpful to transfer to the system a certain knowledge about the expert view on border line cases. The central issue in such pattern recognition systems is the construction of classifiers within vast and poorly understood search spaces, which is a very difficult task. Nonetheless, this process can be greatly enhanced with knowledge about the investigated objects provided by an human expert. We outline a framework for the transfer of such knowledge from the expert and it is shown how to incorporate it into the learning process of a recognition system using methods based on rough mereology [23. Is is also demonstrated how this knowledge acquisition can be conducted in an interactive manner, with a large dataset of handwritten digits as an example.

The outlined research directions create, in our projects, foundations toward understanding the nature of reasoning from measurements to perception. Understanding such reasoning processes is fundamental for developing intelligent systems based on perception logic. A further understanding of perception logic in interdisciplinary projects will make it possible to use discoveries from the different scientific disciplines mentioned in this article for improving the performance of intelligent systems.

\section{Acknowledgment}

The research has been supported by the grant 3 T11C 00226 from Ministry of Scientific Research and Information Technology of the Republic of Poland.

\section{References}

1. J.R. Anderson, C. Lebiere, The Atomic Components of Thought, Mahwah, NJ: Lawrence Erlbaum, 1998.

2. Y. Bar-Yam, Dynamics of Complex Systems, Addison Wesley, 1997.

3. L.W. Barsalou, "Perceptual symbol systems," Behavioral and Brain Sciences 22: 577-660 1999.

4. J. Bazan, A. Skowron, "Classifiers based on approximate reasoning schemes," In: B. Dunin-Keplicz, A. Jankowsk, A. Skowron, and M. Szczuka (eds.), Monitoring, Security, and Rescue Tasks in Multiagent Systems MSRAS, Advances in Soft Computing, Springer, Heidelberg, pp. 191-202, 2005.

5. J. Bazan, J. Peters, A. Skowron, "Behavioral pattern identification through rough set modelling," Proceedings of RSFDGrC 2005, Regina, Canada, September 1-3, 2005 (to appear).

6. S. Behnke, Hierarchical Neural Networks for Image Interpretation, LNCS 2766, Springer, Heidelberg, 3003.

7. E. Bonabeau, M. Dorigo, and G. Theraulaz, Swarm Intelligence. From Natural to Artificial Systems, Oxford University Press, UK, 1999.

8. L. Breiman, "Statistical Modeling: The Two Cultures," Statistical Science 16(3): 199-231, 2001. 
9. F.M. Brown, Boolean Reasoning, Kluwer Academic Publishers, Dordrecht, 1990.

10. R.O. Duda, P.E. Hart, and D.G. Stork, Pattern Classification, Wiley, New York, 2001.

11. B. Dunin-Keplicz, A. Jankowski, A. Skowron, and M. Szczuka (eds.), Monitoring, Security, and Rescue Tasks in Multiagent Systems MSRAS, Advances in Soft Computing, Springer, Heidelberg, 2005.

12. M. Fahle, T. Poggio (eds.), Perceptual Learning, MIT Press, Cambridge, 2002.

13. G. Frege, Grundlagen der Arithmetik 2, Verlag von Herman Pohle, Jena, 1893.

14. J.H. Friedman, T. Hastie, and R. Tibshirani, The Elements of Statistical Learning: Data Mining, Inference, and Prediction, Springer-Verlag, Heidelberg, 2001.

15. M. Gell-Mann, The Quark and the Jaguar - Adventures in the Simple and the Complex, Little, Brown and Co., London, 1994.

16. S. Harnad (ed.), Categorical Perception: The Groundwork of Cognition, Cambridge University Press, New York, 1987.

17. W. Kloesgen, J. Żytkow (eds.), Handbook of Knowledge Discovery and Data Mining, Oxford University Press, 2002.

18. J. Łukasiewicz, "Die Logischen grundlagen der Wahrscheinlichkeitsrechnung," Kraków, 1913, in: L. Borkowski (ed.), Jan Eukasiewicz - Selected Works, North Holland Publishing Company, Amsterdam, London, Polish Scientific Publishers, Warsaw, 1970.

19. S. Leśniewski: "Grungzüge eines neuen Systems der Grundlagen der Mathematik", Fundamenta Matemaicae XIV: 1-81, 1929.

20. M. Luck, P. McBurney, and Ch. Preist, Agent Technology: Enabling Next Generation Computing: A Roadmap for Agent Based Computing, AgentLink 2003.

21. A. Newell, Unified Theories of Cognition, Harvard University Press, Cambridge, MA, 1990.

22. S. Hoa Nguyen, J. Bazan, A. Skowron, and H. Son Nguyen, "Layered learning for concept synthesis," Transactions on Rough Sets I, LNCS3100, Springer, Heidelberg, 2004,187-208.

23. T. T. Nguyen, A. Skowron, "Rough set approach to domain knowledge approximation," In: G. Wang, Q. Liu, Y. Yao, and A. Skowron (eds.), Proceedings of the 9th International Conference: Rough Sets, Fuzzy Sets, Data Mining, and Granular Computing, RSFDGRC'03, Chongqing, China, Oct 19-22, 2003, LNCS 2639, pp. 221-228, Springer Verlag, Heidelberg, 2003.

24. R.W. Paine, S. Grossberg, , and A.W.A. Van Gemmert: "A quantitative evaluation of the AVITEWRITE model of handwriting learning," Human Movement Science (in press), 2004.

25. S.K. Pal, L. Polkowski, and A. Skowron (eds.) Rough-Neural Computing: Techniques for Computing with Words, Cognitive Technologies series, Springer-Verlag, Heidelberg, Germany, 2004.

26. S.K. Pal, A. Skowron (eds.), Rough Fuzzy Hybridization: A New Trend in DecisionMaking, Springer-Verlag, Singapore, 1999.

27. Z. Pawlak, Rough Sets: Theoretical Aspects of Reasoning about Data, Kluwer Academic Publishers, Dordrecht, The Netherlands, 1991.

28. J.F. Peters, "Rough ethology: Towards a biologically-inspired study of collective behavior in intelligent systems with approximation spaces," Transactions on Rough Sets III: 153-174, LNCS 3400, springer, Heidelberg, 2005.

29. J.F. Peters, A. Skowron, P. Synak, and S. Ramanna, "Rough sets and information granulation," In: T.B. Bilgic, D. Baets, and O. Kaynak (eds.), Tenth International Fuzzy Systems Association World Congress IFSA, Istanbul, Turkey, June 30 - July 2, 2003, LNAI 2715, Springer-Verlag, Heidelberg, pp.370-377, 2003. 
30. T. Poggio, S. Smale, "The mathematics of learning: Dealing with data," Notices of the AMS 50(5): 537-544, 2003.

31. L. Polkowski, A. Skowron, "Rough mereology: A new paradigm for approximate reasoning," International Journal of Approximate Reasoning 15(4): 333-365, 1996.

32. A. Skowron, "Rough sets in KDD" (plenary lecture), In: Z. Shi, B. Faltings, and M. Musen (eds.), 16-th World Computer Congress (IFIP'2000): Proceedings of Conference on Intelligent Information Processing (IIP'2000), Publishing House of Electronic Industry, Beijing, pp. 1-17, 2000.

33. A. Skowron, "Toward intelligent systems: Calculi of information granules," Bulletin of the International Rough Set Society 5(1-2): 9-30, 2001.

34. Skowron, A., "Approximate reasoning in distributed environments," In: Zhong, N., Liu, J. (Eds.), Intelligent Technologies for Information Analysis, Springer, Heidelberg, pp. 433-474, 2004.

35. A. Skowron, J. Stepaniuk, "Information granules and rough-neural computing," In: S.K. Pal, L. Polkowski, and A. Skowron (eds.), Rough-Neural Computing: Techniques for Computing with Words, Cognitive Technologies series, Springer-Verlag, Heidelberg, Germany, pp. 43-84, 2004.

36. A. Skowron, P. Synak, "Complex patterns," Fundamenta Informaticae 60(1-4): 351-366, 2004.

37. A. Skowron, P.Synak, "Reasoning in information maps," Fundamenta Informaticae 59(2-3): 241-259, 2004.

38. Staab, S., Studer, R., (Eds.): Handbook on Ontologies. International Handbooks on Information Systems, Springer, Heidelberg, 2004.

39. P. Stone, Layered Learning in Multi-Agent Systems: A Winning Approach to Robotic Soccer, MIT Press, Cambridge, 2000.

40. C. Urmson, et al., "High speed navigation of unrehearsed terrain: Red team technology for Grand Challenge 2004", Report CMU-RI-TR-04-37, The Robotics Institute, Carnegie Mellon University, 2004.

41. V. Vapnik, Statistical Learning Theory, Wiley, New York, 1998.

42. L.A. Zadeh, "Fuzzy sets," Information and Control 8: 338-353, 1965.

43. L.A. Zadeh, "A new direction in AI: Toward a Computational Theory of Perceptions," AI Magazine 22(1):73-84, 2001.

44. RoboCup: www.robocup.org

45. Road simulator: logic.mimuw.edu.pl/ bazan/simulator

46. RSES: logic.mimuw.edu.pl/ rses/

47. WITAS: www.ida.liu.se/ext/witas/ 